\title{
Smart approaches for assessing free-living energy expenditure following identification of types of physical activity
}

Citation for published version (APA):

Plasqui, G. (2017). Smart approaches for assessing free-living energy expenditure following identification of types of physical activity. Obesity Reviews, 18(S1), 50-55. https://doi.org/10.1111/obr.12506

Document status and date:

Published: 01/02/2017

DOI:

10.1111/obr.12506

Document Version:

Publisher's PDF, also known as Version of record

Document license:

Taverne

Please check the document version of this publication:

- A submitted manuscript is the version of the article upon submission and before peer-review. There can be important differences between the submitted version and the official published version of record.

People interested in the research are advised to contact the author for the final version of the publication, or visit the DOI to the publisher's website.

- The final author version and the galley proof are versions of the publication after peer review.

- The final published version features the final layout of the paper including the volume, issue and page numbers.

Link to publication

\footnotetext{
General rights rights.

- You may freely distribute the URL identifying the publication in the public portal. please follow below link for the End User Agreement:

www.umlib.nl/taverne-license

Take down policy

If you believe that this document breaches copyright please contact us at:

repository@maastrichtuniversity.nl

providing details and we will investigate your claim.
}

Copyright and moral rights for the publications made accessible in the public portal are retained by the authors and/or other copyright owners and it is a condition of accessing publications that users recognise and abide by the legal requirements associated with these

- Users may download and print one copy of any publication from the public portal for the purpose of private study or research.

- You may not further distribute the material or use it for any profit-making activity or commercial gain

If the publication is distributed under the terms of Article $25 \mathrm{fa}$ of the Dutch Copyright Act, indicated by the "Taverne" license above, 


\title{
Review
}

\section{Smart approaches for assessing free-living energy expenditure following identification of types of physical activity}

\author{
G. Plasqui
}

Department of Human Biology and Movement Sciences, NUTRIM School of Nutrition and

Translational Research in Metabolism,

Maastricht University Medical Centre +,

Maastricht, The Netherlands

Received 28 November 2016; accepted 1

December 2016

Address for correspondence: G Plasqui, Department of Human Biology and Movement Sciences, Maastricht University, PO Box 616, 6200 MD Maastricht, The Netherlands. E-mail: g.plasqui@maastrichtuniversity.nl

\begin{abstract}
Summary
Accurate assessment of physical activity and energy expenditure has been a research focus for many decades. A variety of wearable sensors have been developed to objectively capture physical activity patterns in daily life. These sensors have evolved from simple pedometers to tri-axial accelerometers, and multi sensor devices measuring different physiological constructs. The current review focuses on how activity recognition may help to improve daily life energy expenditure assessment. A brief overview is given about how different sensors have evolved over time to pave the way for recognition of different activity types. Once the activity is recognized together with the intensity of the activity, an energetic value can be attributed. This concept can then be tested in daily life using the independent reference technique doubly labeled water. So far, many studies have been performed to accurately identify activity types, and some of those studies have also successfully translated this into energy expenditure estimates. Most of these studies have been performed under standardized conditions, and the true applicability in daily life has rarely been addressed. The results so far however are highly promising, and technological advancements together with newly developed algorithms based on physiological constructs will further expand this field of research.
\end{abstract}

Keywords: Accelerometer, obesity, sedentary behavior.

\section{Introduction}

In contrast to our ancestors hunter gatherers, modern day society is less and less dependent on physical activity to obtain food. Running on a treadmill in a climate controlled room to deliberately waste energy is probably not something cavemen would have anticipated as their future. Yet, given the well-known health effects of regular physical activity, it is essential to stimulate physical activity and decrease sedentary time in the population (1-3). In order to do so, valid techniques are necessary that (i) can reliably measure physical activity and all its different components (intensity, duration, type) under unrestrained conditions of daily life and (ii) can be employed to stimulate physical activity by objective and continuous monitoring and feedback on activity behaviour. Wearable sensors have rapidly evolved over the past two decades. Smart approaches to assess daily life physical activity behaviour and also energy expenditure (EE) will continue to advance quickly in the near future. Dozens of activity monitors can be found in the scientific literature (4-6), and on top of that dozens more commercial devices appear on the market at unprecedented rates. These fast developments make it hard to keep track of the different sensors, the (physiological) constructs they try to measure and the eventual validity of the device to actually quantify the outcome parameter of interest, i.e. daily physical activity.

\section{Defining physical activity}

The most well-known definition of physical activity that is quoted in the literature is the one of Caspersen et al.: 'Physical activity can be defined as any bodily movement, produced by skeletal muscles, that results in energy 
expenditure' (7). This definition is elegant because of its simplicity and at the same time the essential information it contains. First, within the definition, a distinction is already made between two commonly used approaches to express physical activity, namely as body movement or as EE. At the same time, both are linked, saying that if you measure any body movement for example with an accelerometer, this will always lead to EE that for example can be measured by indirect calorimetry. Second, by defining physical activity as any bodily movement, it inherently means that all activities, no matter whether they are sedentary, light, moderate or high intensity are all covered by the term 'physical activity'.

When referring to physical activity within this paper, I consider this term to represent the above definition.

\section{Measuring physical activity}

As stated above, physical activity is mostly expressed either in terms of body movement or as EE. The most common activity monitors use accelerometry to assess body movement. Other sensors are now commonly added to the accelerometer to measure additional physiological constructs, such as heart rate, skin temperature, galvanic skin response or non-physiological constructs such as geographic location by global positioning system (8-10). These are often added to try to improve the estimation of EE as the main parameter of interest, especially when studying physical activity from an energy balance perspective. Nevertheless, it should be noted that also body movement obtained from raw accelerometer output is a highly valuable outcome parameter, regardless of whether or not it is translated to a measure of EE. That is, provided that the accelerometer output truly represents a good measurement of body movement and does not represent artefacts or noise. When trying to obtain a measure of free-living physical activity in all its different varieties, this is not a simple task to perform.

\section{The basic concepts of accelerometry to assess body movement}

\section{Different types of accelerometers}

Accelerometers have evolved from simple pedometers, usually with a small metal ball sliding up and down, into more advanced electronic sensors. Different types of electronic sensors are available, and the type of sensor also determines some of the characteristics. For example, about 20 years ago, Bouten et al. described an accelerometer based on piezo-resistive sensors (11), with the advantage that these sensors also detect the gravitational field, i.e. static accelerations, and hence can be used to determine postures. Because these sensors required a constant power source, the battery unit accompanying the accelerometer was large and relatively heavy, decreasing wearability. Therefore, many accelerometers thereafter used piezo-electric elements that do not require a constant power source and consequently were a lot smaller and allowed enough battery power to operate over several weeks (e.g. $(12,13))$. However, these were only capable of measuring dynamic accelerations. Modern day accelerometers are now often capacitive sensors or micro electro-mechanical systems, which are able to measure both static and dynamic accelerations (e.g. ActivPAL (14), Genea (15), Tracmor $\left.{ }_{D}(16)\right)$. Together with technological advancements in memory storage and battery power, these sensors are small, lightweight and capable of measuring over several weeks, opening up the opportunities for posture and activity type recognition.

\section{Accelerometer output-body movement}

Accelerometer output is very often expressed in counts. In general, a count is the rectified acceleration signal $|\mathrm{x}|$ integrated over a certain amount of time (e.g. $1 \mathrm{~min}$ ):

$$
\text { Count }=\int_{0}^{\mathrm{t} 1}|\mathrm{x}| \mathrm{dt} \text {. }
$$

Most current accelerometers are tri-axial, measuring acceleration in the vertical, antero-posterior and medio-lateral direction of the body. It was previously shown that adding the latter two axes to the vertical axis does improve the explained variation in EE (13).

When a tri-axial accelerometer is used, the output is sometimes the sum of counts over the three axes:

$$
\text { Sum }=\int_{0}^{\mathrm{t} 1}|\mathrm{x}| \mathrm{dt}+\int_{0}^{\mathrm{t} 1}|\mathrm{y}| \mathrm{dt}+\int_{0}^{\mathrm{t} 1}|\mathrm{z}| \mathrm{dt}
$$

or the vector magnitude:

Vector magnitude $=\sqrt{ }\left(\mathrm{x}^{2}+\mathrm{y}^{2}+\mathrm{z}^{2}\right)$

One of the main problems is that this output is not comparable between devices, because different activity monitors contain different accelerometers with a different sensitivity and frequency response and also the sampling frequency differs between different devices. In addition, low- and highpass filters are often used to try to separate the acceleration signal arising from human movement from 'noise' (17).

\section{Wearing position}

In general, the traditional wearing position of an accelerometer was the lower back or on the hip. The reason is that this site is as close as possible to the center of mass of the body. Every recorded acceleration therefore represents an acceleration of body mass, which will have the highest impact on EE. Nevertheless, even when located at the waist level, differences in position, e.g. lower back versus hip, may still have an impact on the recorded accelerations (18). Therefore, standardization of the wearing location is highly important, and care should be taken to attach the sensor as tight as possible to the body 
(18). When an accelerometer is positioned somewhere closer to the extremities, such as the wrist, only a small mass is being moved, and hence the relation between recorded accelerations and EE will be different (19).

Depending on the required output, other wearing locations have been used like the thigh, chest, wrist, ankle and foot. For example, the ActivPAL (PAL Technologies Ltd, Glasgow, UK) is worn on the thigh as the position of the sensor in the gravitational field can then be used for classification of sedentary (lying, sitting) versus standing and ambulatory activities (20).

In order to perform more extensive activity recognition, a variety of different sensor locations have been used, and often several sensors were worn simultaneously at different sites (for an overview please refer to Attal et al. (21)). Combining multiple sensors may improve classification accuracy, but limits wearability.

\section{Energy expenditure in daily life as a measure of physical activity}

Doubly labeled water (DLW) is the gold standard to assess total daily energy expenditure (TDEE). This technique uses the stable isotopes deuterium and oxygen-18 to obtain a measure of $\mathrm{CO}_{2}$ production over a period of 1-3 weeks in daily life. After a dose of water that contains both stable isotopes is consumed, deuterium will distribute evenly over the total body water pool whereas oxygen-18 will be in constant isotopic equilibrium between the body water and dissolved $\mathrm{CO}_{2}$. Hence, over time, deuterium will be eliminated from the body as water, and oxygen-18 will be eliminated as both water and $\mathrm{CO}_{2}$. The difference in elimination rates of the two isotopes is then a measure of $\mathrm{CO}_{2}(22,23)$.

The DLW technique is an elegant methodology as it only requires subjects to collect some urine samples (e.g. seven samples over a 2-week period according to the Maastricht protocol (24)) and hence does not interfere in any way with habitual daily activity. The disadvantage is that a measure of TDEE is obtained over one or two weeks with no information about daily activity patterns.

Expressing physical activity as EE requires a measure of TDEE as well as a measurement or estimate of basal metabolic rate (BMR). Physical activity is then commonly expressed as (i) activity-related energy expenditure, which is TDEE minus BMR minus diet-induced thermogenesis, or (ii) as the physical activity level, which is TDEE divided by BMR.

\section{Validating wearable sensors}

In order to say that a wearable sensor is capable of accurately measuring free-living physical activity, validity needs to be tested in daily life versus an independent reference technique. Given the wide variety of different activities performed under unconstrained conditions, little independent reference techniques are available. Direct observation over longer periods of time is not an option because this is an invasion of privacy and has an enormous influence on the activity behaviour itself. To validate activity monitors for their ability to perform activity recognition, one is bound to laboratory conditions or possibly direct observation over shorter periods of time.

The only currently accepted gold standard to evaluate physical activity in daily life is DLW assessed EE. Even though body movement and EE are different constructs, given the above stated definition of physical activity, body movement assessed with an accelerometer should always correlate with activity-related EE as assessed with DLW (in combination with a measurement or estimate of BMR).

\section{Physical activity recognition to improve free- living energy expenditure assessment}

\section{Activity recognition-basic principle}

When accelerometer output is expressed as counts or vector magnitude, the problem arises that the relation between counts and EE will differ depending on the type of activity performed (25-27). For example, let us consider two common daily life activities, i.e. cycling and walking. When an accelerometer is located at the lower back (or hip, or chest), accelerometer output (counts) will increase to a certain extent with increasing cycling intensities and hence increasing EE. When walking is performed, counts will increase to a larger extent with increasing intensities than was the case with cycling (Fig. 1). Activity recognition may provide a solution to this problem, as first the type of activity can be defined as well as the intensity and for each activity a different EE value can be assigned.
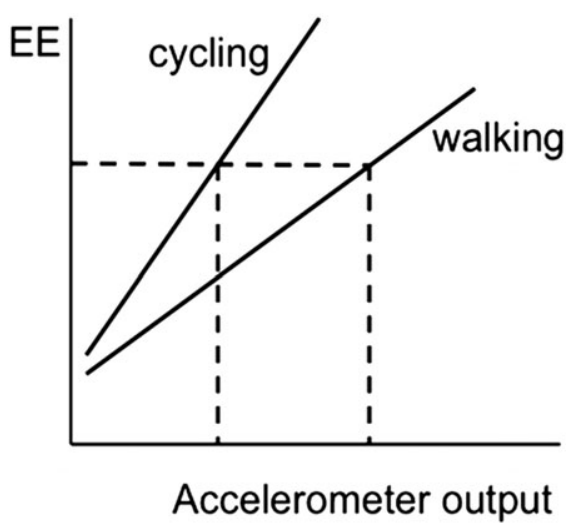

Figure 1 Illustration of a different relationship between accelerometer output (usually counts) and energy expenditure when different activities are being performed. 
Given the huge amount of different activities humans perform in daily life, activity recognition outside laboratory conditions is a challenge. Often, different activities to be recognized are classified into different activity states, i.e. static or postures (e.g. lying, sitting, standing), transitions (e.g. sitting to standing) and dynamic (e.g. walking, cycling). Over the past two decades, many different analytical techniques have been developed for activity classification, from decision trees to more complex artificial neural networks or hidden Markov models. As this review is focused on assessing free-living EE, a description of these computational techniques is beyond the scope of this paper, and the reader is referred to a nice overview provided by Preece et al. (28).

When evaluating different classification approaches, there will be a trade-off between activity recognition accuracy and the amount of sensors involved, sampling frequency, the amount of features to be extracted (e.g. mean, standard deviation, zero crossing rate, frequency peak, etc.) and the selected classifier (e.g. decision tree) as these will all have an impact on computational time, battery power and wearability. This was nicely illustrated in a recent study of Gao et al., who tested the effect of single versus multiple sensors, different sampling frequencies, and different features and classifiers on the recognition accuracy of eight different activities (three static, one dynamic and four transitional) (29). There were only marginal improvements $(1 \%)$ in recognition accuracy by using a sampling frequency above $20 \mathrm{~Hz}$ and no improvements above $50 \mathrm{~Hz}$. They also found the decision tree to be the best out of five different classifiers in terms of a trade-off between accuracy and computational time as it scored only marginally less on accuracy $(96.4 \%)$ compared to an artificial neural network classifier $(96.8 \%)$, where the latter had a computational time (i.e. training time + testing time) of $492 \mathrm{~s}$ versus $4 \mathrm{~s}$ for the decision tree. Using this decision tree, the best multi-sensor system (chest, waist, thigh, side) achieved a recognition accuracy of $96.4 \%$ versus $92.8 \%$ for the best single sensor system (waist) (29).

\section{Activity recognition and energy expenditure}

In 2003, Zhang et al. introduced a new Intelligent Device for Energy Expenditure and Activity (IDEEA; MiniSun, Fresno, CA), a wearable system that was a combination of several sensors located at different parts of the body, connected by flexible wiring aimed to perform activity recognition (30). The IDEEA was able to recognize 32 different types of physical activity including lying, sitting, standing and gait, even at different gait speeds, with an overall identification accuracy of $98.7 \%$. In two laboratory-based protocols (50-min facemask and 23-h respiration chamber), the IDEEA also performed well in estimating EE by first recognizing the type and intensity of an activity and then applying a 'known' EE value corresponding that activity (31). The IDEEA was later also validated in daily life against DLW assessed TEE (32). When resting metabolic rate and $\mathrm{EE}$ for sitting and standing were calculated (included in manufacturer's software), the IDEEA overestimated TEE by 2.61 (SD 1.10) $\mathrm{MJ} \mathrm{d}^{-1}$. When RMR and EE for sitting and standing were measured instead of calculated, the overestimation in TEE was reduced to 1.35 (SD 1.01) $\mathrm{MJ} \mathrm{d}^{-1}$ (32). It should be kept in mind that the error in EE assessment can not only arise from recognizing the correct type and intensity of activity but also from the energetic value assigned to it. For some of the activity types that the IDEEA can recognize, a corresponding EE value was measured by a metabolic cart or room calorimeter, and for others the compendium of physical activities (33) was used (31). Regardless of whether or not the obtained accuracy is considered sufficient, the authors also indicated that in only $57 \%$ of the days, data was collected over 15 hours or more. The IDEEA was also used in the DIOGenes dietary intervention study in overweight and obese subjects (34). There the number of valid days ( $\geq 15$-h data) was only $22 \%$, which increased to $44 \%$ when $\geq 12 \mathrm{~h} \mathrm{~d}^{-1}$ of valid data was used as a criterion (32). This pointed out that such a device, with multiple sensors and a data collection unit has good applicability in laboratory settings or even in daily life for research purposes in highly motivated subjects, but will not be generally applicable in larger populations to estimate and/or stimulate daily life EE. Nevertheless, the concept of accurately recognizing types of activities as well as intensities and then applying an energetic value to the activity performed was proven to be valuable.

The above studies indicate that multiple sensors combined may increase accuracy but will, at least at this moment, substantially decrease wearability. Bonomi et al. further explored the idea of improving EE prediction by applying activity recognition with a single tri-axial accelerometer $(8 \times 3.5 \times 1 \mathrm{~cm}, 34.8 \mathrm{~g}$, sample frequency $20 \mathrm{~Hz}$, decision tree) located at the lower back under free-living conditions (35). EE was simultaneously measured using DLW. Six different activity types were used for classification, i.e. 'lying', 'sitting or standing', 'active standing', 'walking', 'running' and 'cycling', and a corresponding MET value (36) was assigned to each activity type. Using this approach compared to using simple 'counts' as accelerometer output, the explained variation in TEE increased by $9 \%$ and the standard error of estimate (SEE) decreased from 1.47 to $1.33 \mathrm{MJ} \mathrm{d}^{-1}$ (35).

Activity recognition to improve estimates of EE has great potential, and numerous studies have been performed using standardized activities to test recognition accuracy (21). Apart from the above mentioned studies of Whybrow et al. and Bonomi et al. $(32,35)$, to my knowledge no other true daily life studies have been performed to test validity of 
a system or computational technique to improve estimates of TDEE.

\section{Activity recognition-future directions}

In a recent study of Sasaki et al., activity recognition was performed during a standardized activity protocol as well as under free-living conditions in older adults using direct observation as the reference technique (37). The algorithms that were developed during the laboratory-based protocol performed worse in classifying activity types than the algorithm developed from the free-living data. This is in line with a paper from Gyllensten et al. that the accuracy of activity recognition declines substantially when laboratory developed algorithms are being applied to free-living data (38). This indicates that in future studies, free-living data needs to be included when new machine learning algorithms are being developed to obtain better activity recognition accuracy.

The enormous incline in smartphone users over the past decade has made this an attractive device for continuous activity monitoring. The smartphone usually contains different sensors (e.g. accelerometer, gyroscope, barometric pressure sensor, global positioning system) and has the computational power and connectivity to be used for long-term data collection. The smartphone has the additional advantage that users carry the device on them practically at all times. A disadvantage, however, is that women tend to wear their phone in their handbag. Del Rosario et al. used a smartphone placed in the front pocket to perform free-living activity recognition based on an embedded triaxial accelerometer, triaxial gyroscope and barometric pressure sensor (39). During a free-living period of at least $30 \mathrm{~min}$, older subjects performed a variety of activities they might do in normal daily life, including lying, sitting, standing, walking, walking stairs and so on, while the entire data collection protocol was recorded on video. The classification algorithm based on a decision tree was able to achieve an overall sensitivity of $82 \%$ (39). The same authors report similar results for a younger population (total classification sensitivity $81 \%$ ) (39). Also, Anjum et al. developed an algorithm that was built into a smartphone application and was able to recognize seven different activities (walking, running, climbing stairs, descending stairs, driving, cycling and being inactive) with an average sensitivity of $95 \%$ (40). This indicates the possibilities of smart phones performing real-time activity recognition with good sensitivity. Another advantage of smartphones is that thanks to their connectivity also other sensors located at different locations at the body could be connected to the phone (via bluetooth or wifi).

Little work so far has been performed to investigate the possibility of adding different sensors that measure other (physiological) constructs to improve activity recognition. When performing these studies, it should be acknowledged that adding multiple sensors will demand more computational time and power and may decrease wearability. However, it should also be kept in mind that the rapid technological advances may overcome these problems and that newly developed concepts that are currently not applicable in large populations or outside standardized settings may well be so in the near future.

\section{Conclusion}

The purpose of this review was to provide an overview of how wearable sensors can be applied to improve daily life EE assessment with a special focus on activity recognition. To introduce the subject, I have tried to provide a short overview of how sensors have evolved over time, why EE assessed with DLW is an appropriate reference technique and how activity recognition can help improve the estimation of EE in daily life. From the presented literature, it is clear that many studies have been performed under standardized conditions but very little under truly free-living conditions. Several reasons for that are apparent. First, when performing activity recognition, one would like to validate the methodology against another gold standard that also assesses activity types. Unfortunately, no such technique is available, except for direct or video observation which is clearly not applicable in daily life settings over longer periods of time. DLW measures a different construct, i.e. $\mathrm{EE}$, and may therefore not be the first method of choice to validate activity recognition data. However, when activity recognition is performed in an attempt to better estimate free-living EE, DLW is the best validation technique.

Wearable sensors have already been proven highly valuable in different research domains, also outside physical activity or EE assessment (e.g. fall detection, gait analysis). Together with advancements in sensor technology, new applications will continue to arise in the near future.

\section{Conflict of interest statement}

No conflict of interest was declared.

\section{References}

1. Barengo NC, Hu G, Lakka TA, Pekkarinen $H$, Nissinen A, Tuomilehto J. Low physical activity as a predictor for total and cardiovascular disease mortality in middle-aged men and women in Finland. Eur Heart J 2004; 25: 2204-2211.

2. Hu FB, Li TY, Colditz GA, Willett WC, Manson JE. Television watching and other sedentary behaviors in relation to risk of obesity and type 2 diabetes mellitus in women. JAMA 2003; 289: 1785-1791.

3. Hu G, Qiao Q, Silventoinen K et al. Occupational, commuting, and leisure-time physical activity in relation to risk for Type 2 diabetes in middle-aged Finnish men and women. Diabetologia 2003; 46: 322-329. 
4. Plasqui G, Westerterp KR. Physical activity assessment with accelerometers: an evaluation against doubly labeled water. Obesity (Silver Spring) 2007; 15: 2371-2379.

5. Plasqui G, Bonomi AG, Westerterp KR. Daily physical activity assessment with accelerometers: new insights and validation studies. Obes Rev 2013; 14: 451-462.

6. Westerterp KR. Reliable assessment of physical activity in disease: an update on activity monitors. Curr Opin Clin Nutr Metab Care 2014; 17: 401-406.

7. Caspersen CJ, Powell KE, Christenson GM. Physical activity, exercise, and physical fitness: definitions and distinctions for health-related research. Public Health Rep 1985; 100: 126-131.

8. Butte NF, Wong WW, Adolph AL, Puyau MR, Vohra FA, Zakeri IF. Validation of cross-sectional time series and multivariate adaptive regression splines models for the prediction of energy expenditure in children and adolescents using doubly labeled water. J Nutr 2010; 140: 1516-1523.

9. Johannsen DL, Calabro MA, Stewart J, Franke W, Rood JC, Welk GJ. Accuracy of armband monitors for measuring daily energy expenditure in healthy adults. Med Sci Sports Exerc 2010; 42: 2134-2140.

10. Rodriguez DA, Brown AL, Troped PJ. Portable global positioning units to complement accelerometry-based physical activity monitors. Med Sci Sports Exerc 2005; 37: S572-S581.

11. Bouten CV, Koekkoek KT, Verduin M, Kodde R, Janssen JDA. triaxial accelerometer and portable data processing unit for the assessment of daily physical activity. IEEE Trans Biomed Eng 1997; 44: 136-147.

12. Yngve A, Nilsson A, Sjostrom M, Ekelund U. Effect of monitor placement and of activity setting on the MTI accelerometer output. Med Sci Sports Exerc 2003; 35: 320-326.

13. Plasqui G, Joosen AM, Kester AD, Goris AH, Westerterp KR. Measuring free-living energy expenditure and physical activity with triaxial accelerometry. Obes Res 2005; 13: 1363-1369.

14. Ryan CG, Grant PM, Tigbe WW, Granat MH. The validity and reliability of a novel activity monitor as a measure of walking. Br J Sports Med 2006; 40: 779-784.

15. Esliger DW, Rowlands AV, Hurst TL, Catt M, Murray P, Eston RG. Validation of the GENEA Accelerometer. Med Sci Sports Exerc 2011; 43: 1085-1093.

16. Bonomi AG, Plasqui G, Goris AH, Westerterp KR. Estimation of free-living energy expenditure using a novel activity monitor designed to minimize obtrusiveness. Obesity (Silver Spring) 2010; 18: $1845-1851$.

17. Lowe SA, Olaighin G. Monitoring human health behaviour in one's living environment: a technological review. Med Eng Phys 2014; 36: 147-168.

18. Boerema ST, van Velsen L, Schaake L, Tonis TM, Hermens HJ. Optimal sensor placement for measuring physical activity with a 3D accelerometer. Sensors (Basel) 2014; 14: 3188-3206.

19. Kim DY, Jung YS, Park RW, Joo NS. Different location of triaxial accelerometer and different energy expenditures. Yonsei Med J 2014; 55: 1145-1151.

20. Grant PM, Ryan CG, Tigbe WW, Granat MH. The validation of a novel activity monitor in the measurement of posture and motion during everyday activities. Br J Sports Med 2006; 40: 992-997. 21. Attal F, Mohammed S, Dedabrishvili M, Chamroukhi F, Oukhellou L, Amirat Y. Physical human activity recognition using wearable sensors. Sensors (Basel) 2015; 15: 31314-31338.

22. Schoeller DA, Ravussin E, Schutz Y, Acheson KJ, Baertschi P, Jequier E. Energy expenditure by doubly labeled water: validation in humans and proposed calculation. Am J Phys 1986; 250: R823-R830.

23. Speakman JR. The history and theory of the doubly labeled water technique. Am J Clin Nutr 1998; 68: 932S-938S.

24. Westerterp KR, Wouters L, van Marken Lichtenbelt WD. The Maastricht protocol for the measurement of body composition and energy expenditure with labeled water. Obes Res 1995; 3: 49-57.

25. Crouter SE, Clowers KG, Bassett DR Jr. A novel method for using accelerometer data to predict energy expenditure. $J$ Appl Physiol 2006; 100: 1324-1331.

26. Midorikawa T, Tanaka S, Kaneko K et al. Evaluation of lowintensity physical activity by triaxial accelerometry. Obesity (Silver Spring) 2007; 15: 3031-3038.

27. Bonomi AG, Goris AH, Yin B, Westerterp KR. Detection of type, duration, and intensity of physical activity using an accelerometer. Med Sci Sports Exerc 2009; 41: 1770-1777.

28. Preece SJ, Goulermas JY, Kenney LP, Howard D, Meijer K, Crompton R. Activity identification using body-mounted sensors - a review of classification techniques. Physiol Meas 2009; 30: R1-33.

29. Gao L, Bourke AK, Nelson J. Evaluation of accelerometer based multi-sensor versus single-sensor activity recognition systems. Med Eng Phys 2014; 36: 779-785.

30. Zhang K, Werner P, Sun M, Pi-Sunyer FX, Boozer CN. Measurement of human daily physical activity. Obes Res 2003; 11: $33-40$.

31. Zhang K, Pi-Sunyer FX, Boozer CN. Improving energy expenditure estimation for physical activity. Med Sci Sports Exerc 2004; 36: 883-889.

32. Whybrow S, Ritz P, Horgan GW, Stubbs RJ. An evaluation of the IDEEA activity monitor for estimating energy expenditure. $\mathrm{Br} J$ Nutr 2013; 109: 173-183.

33. Ainsworth BE, Haskell WL, Leon AS et al. Compendium of physical activities: classification of energy costs of human physical activities. Med Sci Sports Exerc 1993; 25: 71-80.

34. Larsen TM, Dalskov S, van Baak M et al. The Diet, Obesity and Genes (Diogenes) Dietary Study in eight European countries - a comprehensive design for long-term intervention. Obes Rev 2010; 11: 76-91.

35. Bonomi AG, Plasqui G, Goris AH, Westerterp KR. Improving assessment of daily energy expenditure by identifying types of physical activity with a single accelerometer. J Appl Physiol 2009; 107: 655-661.

36. Ainsworth BE, Haskell WL, Whitt MC et al. Compendium of physical activities: an update of activity codes and MET intensities. Med Sci Sports Exerc 2000; 32: S498-S504.

37. Sasaki JE, Hickey AM, Staudenmayer JW, John D, Kent JA, Freedson PS. Performance of activity classification algorithms in free-living older adults. Med Sci Sports Exerc 2016; 48: 941-950.

38. Gyllensten IC, Bonomi AG. Identifying types of physical activity with a single accelerometer: evaluating laboratory-trained algorithms in daily life. IEEE Trans Biomed Eng 2011; 58: 2656-2663.

39. Del Rosario MB, Wang K, Wang J et al. A comparison of activity classification in younger and older cohorts using a smartphone. Physiol Meas 2014; 35: 2269-2286.

40. Anjum A, Ilyas MU. Activity recognition using smartphone sensors. IEEE Consumer Communications and Networking Conference: Las Vegas 2013; 914-9. 